\title{
Time-Dependent Transport through a Mesoscopic Structure
}

\author{
Ned S. Wingreen \\ NEC Research Institute, 4 Independence Way, Princeton, NJ 08540 \\ Antti-Pekka Jauho* \\ NORDITA, Blegdamsvej 17, DK-2100 Copenhagen, Denmark \\ Yigal Meir \\ Department of Physics, University of California, Santa Barbara, CA 93106
}

\begin{abstract}
We present a general formulation of the nonlinear, time-dependent current through a small interacting region, where electron energies are changed by timedependent voltages. An exact solution is obtained for the non-interacting case when the elastic coupling to the leads is independent of energy. Temporal phase coherence in a double-barrier tunneling structure produces "ringing" in the response of the current to a voltage pulse, which can be observed experimentally in the dc-current by varying the pulse length in a train of voltage pulses. The nonlinear current due to an ac-bias also shows complex time-dependence.
\end{abstract}

PACS numbers: 73.20.Dx 73.40.Ei 73.40.Gk 73.50.Fq

Typeset Using REVTEX 
The importance of the spatial coherence of electronic wavefunctions is one of the hallmarks of the mesoscopic regime. A panoply of mesoscale phenomena including weaklocalization and weak anti-localization, ${ }^{1}$ Aharonov-Bohm oscillations, ${ }^{2}$ and Universal conductance fluctuations $^{2}$ all rely on the phase coherence of electrons in small structures. Since the traditional experimental probe of these effects is steady-state transport, the role of temporal phase coherence is generally subsumed under the effects of spatial coherence. However, recent experimental progress ${ }^{3-5}$ has opened the door to direct measurement of electronic phase coherence in time.

Interest in the time domain has sprung both from device applications of double-barrier resonant-tunneling structures, ${ }^{3}$ and from possible current standard applications of singleelectron tunneling circuits. ${ }^{4}$ While the interactions among electrons are mean-field like in the three-dimensional resonant-tunneling structures, the low-dimensional confinement of electrons in the single-electron circuits results in strong many-body correlations. A general theory for temporal coherence in these structures must therefore rigorously account for interactions.

In this letter, we first present a general formulation of the nonlinear, time-dependent current through a small interacting region coupled to two non-interacting leads. ${ }^{6} \mathrm{~A}$ general result, Eq. (6), expresses the time-dependent current flowing into the interacting region from one lead in terms of local Green functions. This expression is then used to explore temporal coherence in the response of a mesoscopic system to time-dependent external driving. We focus on a fully nonlinear, exactly solvable example — non-interacting electrons traversing a double-barrier tunneling structure with energy independent coupling to the leads. The temporal coherence of this system is evident in the "ringing" of the current in response to a rectangular pulse of the bias. This ringing can be observed experimentally in the $d c$-current by varying the pulse length in a train of voltage pulses applied to the structure. Similarly, the current flowing in response to an ac-bias displays complex time-dependence, which is reflected in oscillations of the dc-current vs. driving frequency. ${ }^{7}$

The formalism and Hamiltonian are similar to those employed for the steady-state current 
in Ref. 8,

$$
H=\sum_{k, \alpha \in L / R} \epsilon_{k \alpha}(t) \mathbf{c}_{k \alpha}^{\dagger} \mathbf{c}_{k \alpha}+H_{\mathrm{int}}\left[\left\{\mathbf{d}_{n}\right\},\left\{\mathbf{d}_{n}^{\dagger}\right\}, t\right]+\sum_{\substack{k, \alpha \in L / R \\ n}}\left[V_{k \alpha, n}(t) \mathbf{c}_{k \alpha}^{\dagger} \mathbf{d}_{n}+h . c .\right]
$$

where $\mathbf{c}_{k \alpha}^{+}\left(\mathbf{c}_{k \alpha}\right)$ creates (destroys) an electron with momentum $k$ in channel $\alpha$ in either the left $(L)$ or the right $(R)$ lead, and $\left\{\mathbf{d}_{n}^{+}\right\}$and $\left\{\mathbf{d}_{n}\right\}$ form a complete, orthonormal set of single-electron creation and annihilation operators in the interacting region. However, time dependence due to external driving is now explicitly included in the energies of states in the interacting region and in the leads, in the hopping matrix elements, and in the interactions themselves. A central assumption is that interactions between electrons in the leads and between electrons in the leads and in the interacting region can be neglected. Geometrically, the leads must therefore rapidly broaden into large metallic contacts in which interactions are strongly screened. Since transport experiments on mesoscale structures typically satisfy this requirement, the model is directly relevant to experiment. Note that because timedependent potentials must be associated with changes of charge, there will be capacitive currents flowing in the contacts in addition to the currents from the interacting region determined by (11). The contribution of the capacitive currents can be eliminated, however, by measuring only the time-averaged current.

The Keldysh approach ${ }^{9}$ to calculating the current flowing into and out of the interacting region treats the contacts as systems separately in equilibrium in the distant past, possibly with different chemical potentials. Physically, applying a time-dependent bias (electrostatic-potential difference) changes the energies of states in the leads via $\epsilon_{k \alpha}(t)$, without changing their occupations. This preserves the coherent evolution of phase in the leads, $\Psi_{k \alpha}(t) \propto \exp \left[-i \int d t^{\prime} \epsilon_{k \alpha}\left(t^{\prime}\right)\right]$, and produces interference in tunneling between the leads and the interacting region, due to their different time-dependent energies.

The time-dependent current from the left lead into the interacting region is

$$
J_{L}(t)=\frac{2 e}{\hbar} \operatorname{Re}\left\{\sum_{\substack{k, \alpha \in L \\ n}} V_{k \alpha, n}(t) G_{n, k \alpha}^{<}(t, t)\right\}
$$

where in (2) we have just rewritten the expectation of the current matrix element in terms of 
the Keldysh Green function ${ }^{9} G_{n, k \alpha}^{<}\left(t, t^{\prime}\right) \equiv i\left\langle\mathbf{c}_{k \alpha}^{+}\left(t^{\prime}\right) \mathbf{d}_{n}(t)\right\rangle$. Since the Hamiltonian describing the leads is non-interacting, one has the Dyson equation

$$
G_{n, k \alpha}^{<}\left(t, t^{\prime}\right)=\sum_{m} \int d t_{1} V_{k \alpha, m}^{*}\left(t_{1}\right)\left[G_{n, m}^{r}\left(t, t_{1}\right) g_{k \alpha, k \alpha}^{<}\left(t_{1}, t^{\prime}\right)+G_{n, m}^{<}\left(t, t_{1}\right) g_{k \alpha, k \alpha}^{a}\left(t_{1}, t^{\prime}\right)\right]
$$

where $G_{n, m}^{<}\left(t, t^{\prime}\right) \equiv i\left\langle\mathbf{d}_{m}^{+}\left(t^{\prime}\right) \mathbf{d}_{n}(t)\right\rangle$, and $G_{n, m}^{r}\left(t, t^{\prime}\right) \equiv-i \theta\left(t-t^{\prime}\right)\left\langle\left\{\mathbf{d}_{m}^{+}\left(t^{\prime}\right), \mathbf{d}_{n}(t)\right\}\right\rangle$ is the retarded Green function. The time-dependent Green functions in the leads for the uncoupled system, which appear in (3), are given by

$$
\begin{aligned}
& g_{k \alpha, k \alpha}^{<}\left(t, t^{\prime}\right)=i f\left(\epsilon_{k \alpha}^{0}\right) \exp \left[-i \int_{t^{\prime}}^{t} d t_{1} \epsilon_{k \alpha}\left(t_{1}\right)\right] \\
& g_{k \alpha, k \alpha}^{a}\left(t, t^{\prime}\right)=i \theta\left(t^{\prime}-t\right) \exp \left[-i \int_{t^{\prime}}^{t} d t_{1} \epsilon_{k \alpha}\left(t_{1}\right)\right]
\end{aligned}
$$

where $\epsilon_{k \alpha}^{0}$ is the energy of the state $k$, with occupation $f\left(\epsilon_{k \alpha}^{0}\right)$, when the system was prepared in the distant past, and the advanced Green function is related to the retarded Green function by $g^{a}\left(t, t^{\prime}\right)=\left[g^{r}\left(t^{\prime}, t\right)\right]^{*}$. It is convenient in (2) to turn the sum over momentum states $k$ in the leads into an integral over energies, and to define the elastic coupling between the leads and the states in the interacting region via

$$
\left[\Gamma^{L}\left(\epsilon, t^{\prime}, t\right)\right]_{m, n}=2 \pi \sum_{\alpha \in L} \rho_{\alpha}(\epsilon) V_{\alpha, n}(\epsilon, t) V_{\alpha, m}^{*}\left(\epsilon, t^{\prime}\right) \exp \left[i \int_{t^{\prime}}^{t} d t_{1} \Delta_{\alpha}\left(\epsilon, t_{1}\right)\right]
$$

where $\rho_{\alpha}(\epsilon)$ is the density of states in channel $\alpha$ and the energy, $\epsilon_{k \alpha}(t)$, of each state in the leads is separated into a constant part $\epsilon_{k \alpha}^{0}=\epsilon$ and a time-dependent part $\Delta_{\alpha}(\epsilon, t)$. Rewriting the current in terms of the elastic coupling, and using matrix notation for the level indices in the interacting region, we find

$$
J_{L}(t)=-\frac{2 e}{\hbar} \int_{-\infty}^{t} d t^{\prime} \int \frac{d \epsilon}{2 \pi} \operatorname{Im} \operatorname{Tr}\left\{\mathrm{e}^{i \epsilon\left(t-t^{\prime}\right)} \boldsymbol{\Gamma}^{L}\left(\epsilon, t^{\prime}, t\right)\left[\mathbf{G}^{<}\left(t, t^{\prime}\right)+f_{L}(\epsilon) \mathbf{G}^{r}\left(t, t^{\prime}\right)\right]\right\}
$$

An analogous expression applies for the current flowing in from the right lead.

Eq. (6) is the central formal result of this work. The strong resemblance to the steadystate result in Ref. (8) means that the time-dependent problem is not significantly harder than the time-independent one. For a range of mesoscopic systems, a rigorous formula for the steady-state current is a useful tool, and we believe (6) will be a useful tool to 
study dynamics. While interacting quantum transport is addressable with (6), we use it here to explore dynamics in an exactly solvable, non-interacting system corresponding to a quantum-well structure.

In general, if interactions are neglected, the retarded and advanced Green functions are given by standard Dyson equations, ${ }^{10}$ and the Keldysh Green function in (6) is related to them via

$$
\mathbf{G}^{<}\left(t, t^{\prime}\right)=i \int d t_{1} \int d t_{2} \mathbf{G}^{r}\left(t, t_{1}\right)\left[\sum_{L, R} \int \frac{d \epsilon}{2 \pi} e^{i \epsilon\left(t_{2}-t_{1}\right)} f_{L / R}(\epsilon) \boldsymbol{\Gamma}^{L / R}\left(\epsilon, t_{1}, t_{2}\right)\right] \mathbf{G}^{a}\left(t_{2}, t^{\prime}\right) .
$$

A tractable, non-interacting example of particular interest corresponds to a double-barrier tunneling structure containing a single resonant level, $\epsilon_{0}(t)=\epsilon_{0}+\Delta(t)$, with energyindependent coupling to two leads,

$$
\Gamma_{L / R}\left(t^{\prime}, t\right)=\Gamma_{L / R} \exp \left[i \int_{t^{\prime}}^{t} d t_{1} \Delta_{L / R}\left(t_{1}\right)\right]
$$

In (8), the time dependence in the leads is restricted to a rigid shift of all states in the left(right) lead by $\Delta_{L(R)}(t)$. Within this framework, application of a voltage bias corresponds to a shift of energies in one lead with respect to the other, and generally a shift of the resonant level as well. For energy-independent coupling to the leads, the retarded Green function for the resonant level is independent of energy shifts $\Delta_{L / R}(t)$ in the leads, and is given by ${ }^{11}$ $G^{r}\left(t, t^{\prime}\right)=\exp \left[-\Gamma\left(t-t^{\prime}\right) / 2\right] g^{r}\left(t, t^{\prime}\right)$, where $g^{r}\left(t, t^{\prime}\right)$ is the retarded Green function for the uncoupled level,

$$
g^{r}\left(t, t^{\prime}\right)=-i \theta\left(t-t^{\prime}\right) \exp \left[-i \int_{t^{\prime}}^{t} d t_{1} \epsilon_{0}\left(t_{1}\right)\right]
$$

and where $\Gamma \equiv \Gamma_{L}+\Gamma_{R}$ is the total elastic coupling to the leads.

The resulting expression for the retarded Green function of the resonant level, $G^{r}\left(t, t^{\prime}\right)$, can be used in (17) to generate the Keldysh Green function, $G^{<}\left(t, t^{\prime}\right)$, which contains all the information concerning non-equilibrium occupations. Physical quantities such as the occupancy of the level and the currents through the barriers can then be expressed directly in terms of $G^{<}\left(t, t^{\prime}\right)$, or, more conveniently via (7), in terms of $G^{r}\left(t, t^{\prime}\right)$ and the occupation 
functions in the leads. The occupancy of the level, $n(t)=-i G^{<}(t, t)$, and the current, $J_{L / R}$ from (6), are given by:

$$
\begin{array}{r}
n(t)=\sum_{L / R} \Gamma_{L / R} \int \frac{d \epsilon}{2 \pi} f_{L / R}(\epsilon)\left|A_{L / R}(\epsilon, t)\right|^{2} \\
J_{L / R}(t)=-\frac{e}{\hbar} \Gamma_{L / R}\left[n(t)+\int \frac{d \epsilon}{\pi} f_{L / R}(\epsilon) \operatorname{Im}\left\{A_{L / R}(\epsilon, t)\right\}\right]
\end{array}
$$

where we have defined

$$
A_{L / R}(\epsilon, t)=\int_{-\infty}^{t} d t_{1} \exp \left[i \int_{t_{1}}^{t} d t_{2}\left(\epsilon+\Delta_{L / R}\left(t_{2}\right)\right)\right] G^{r}\left(t, t_{1}\right)
$$

In the time-independent case, $A_{L / R}(\epsilon, t)$ is the Fourier transform of the retarded Green function, and one finds the usual result for the resonant-tunneling current,

$$
J=\frac{e}{h} \int d \epsilon\left[f_{L}(\epsilon)-f_{R}(\epsilon)\right] \frac{\Gamma_{L} \Gamma_{R}}{\left(\epsilon-\epsilon_{0}\right)^{2}+(\Gamma / 2)^{2}} .
$$

While arbitrary time dependence of both the bias and level energy can be addressed via Eqs. (912), we have chosen to consider the responses to a rectangular pulse and to an acbias as examples of experimental relevance. As discussed above, an important experimental caveat is that the time-dependent current flowing in the contacts will include capacitive contributions from the accumulation and depletion layers on either side of the tunneling barriers. ${ }^{12}$ It is possible, however, to measure exclusively the current flowing through the barriers by measuring the time-averaged current, since the dc-current must be uniform throughout the structure.

From Eq. (12), one finds for a rectangular pulse of duration $s$ starting at $t=0$,

$$
A_{L / R}(\epsilon, t)=\frac{\epsilon-\epsilon_{0}+i \Gamma / 2-\left(\Delta-\Delta_{L / R}\right)\left[1-e^{i\left(\epsilon-\epsilon_{0}+i \Gamma / 2\right)(t-s)}\left(1-e^{i\left(\epsilon-\epsilon_{0}-\Delta+\Delta_{L / R}+i \Gamma / 2\right) s}\right)\right]}{\left(\epsilon-\epsilon_{0}+i \Gamma / 2\right)\left(\epsilon-\epsilon_{0}-\Delta+\Delta_{L / R}+i \Gamma / 2\right)} .
$$

Expression (14) for $A_{L / R}(\epsilon, t)$ applies for times, $t>s$, after the pulse has ended. For times during the pulse, $0<t<s$, one should replace $s$ by $t$, which follows from causality. In 
Fig. 1a, the current flowing through the barriers is plotted for a voltage pulse of duration $s=3 \hbar / \Gamma$ (dashed curve). Before the pulse, the chemical potentials $\mu_{L / R}$ and the level energy $\epsilon_{0}$ are equal so the current is zero. During the pulse, energies in the left lead are increased by $\Delta_{L}=10 \Gamma$ and the energy of the resonant level is raised by $\Delta=5 \Gamma$, appropriate for a symmetric structure (inset, Fig. 1b). (The choice $\Delta_{R}=0$ is arbitrary since only the relative shifts of energy are significant.) Initially the current through each barrier grows proportional to $t \log (1 / t),{ }^{13}$ and then oscillates with a period $\Delta t=2 \pi \hbar /\left|\left(\mu_{L / R}+\Delta_{L / R}\right)-\left(\epsilon_{0}+\Delta\right)\right|(\simeq 1.26$ in Fig. 1a). The time scale for the decay of the oscillations is the resonance lifetime $\hbar / \Gamma$, i. e., the time during which the states comprising the resonance remain in phase and hence contribute constructively to the current oscillations. For the case shown in Fig. 1a, the occupancy remains fixed at $n=0.5$ by symmetry, and so the currents through the two barriers must be equal. The "ringing" in the current is a consequence of the different phase evolution of the resonant level and of the states in the leads. Such phase coherence is explicitly absent in the calculation of time-dependent resonant-tunneling currents by Chen and Ting, ${ }^{14}$ and so they find only exponential relaxation following an abrupt change of bias.

To observe the "ringing" experimentally, a series of pulses such as that of Fig. 1a could be applied to a tunneling structure and the dc-current measured as a function of pulse duration. ${ }^{15}$ In Fig. 1b, the derivative of the dc-current with respect to pulse length is plotted, normalized by the repeat time, $\tau$, between pulses. For pulse lengths, $s$, of the order of the resonance lifetime, $\hbar / \Gamma$, the derivative of the dc-current mimics closely the timedependent current following the pulse, and, likewise, asymptotes to the steady-state current at the new voltage. We assume that the pulse duration, of order a typical resonance lifetime, $\hbar / \Gamma \sim 1-1000 \mathrm{ps}$, will be much shorter than the time between pulses, so that pulses will not interfere.

Instead of applying a bias pulse, it may be experimentally more practical to apply an ac-bias to the tunneling structure. ${ }^{3}$ From Eq. (12), one finds for an ac-potential,

$$
A_{L / R}(\epsilon, t)=\exp \left[-i \frac{\Delta-\Delta_{L / R}}{\hbar \omega} \sin (\omega t)\right] \sum_{k=-\infty}^{\infty} J_{k}\left(\frac{\Delta-\Delta_{L / R}}{\hbar \omega}\right) \frac{\exp (i k \omega t)}{\epsilon-\epsilon_{0}-k \hbar \omega+i \Gamma / 2},
$$


where $\Delta_{L / R}(t)=\Delta_{L / R} \cos (\omega t), \Delta(t)=\Delta \cos (\omega t)$, and $J_{k}$ is the $k^{t h}$ order Bessel function. In Fig. 2a, the current is plotted for an ac-potential of frequency $\omega=2 \Gamma / \hbar$. While the current has the same period, $2 \pi / \omega$, the complex time-dependence inside each period is similar to the "ringing" response to a pulse shown in Fig. 1. One consequence of this complex harmonic structure is that for temperatures $k_{B} T<\hbar \omega$ the time-averaged current, $J_{\mathrm{dc}}$, oscillates as a function of period, as shown in Fig. 2b. An oscillation occurs whenever a photon-assisted resonant-tunneling peak aligns with one chemical potential, $\epsilon_{0}+k \hbar \omega=\mu_{L / R}$, giving oscillations periodic in $1 / \omega \cdot{ }^{7}$ Direct measurement of the ac-current is complicated by the capacitive contributions from the leads, but we expect the structure shown in Fig. 2a to survive.

In conclusion, a general formula (6) for the time-dependent current through an interacting mesoscopic region has been obtained in terms of local Green functions. While for the interacting case only approximate Green function solutions may be available, for a single non-interacting level with energy-independent coupling to two leads an exact solution has been obtained (9-12). This case corresponds experimentally to resonant tunneling through a double-barrier structure. We find that temporal coherence of electrons tunneling through the resonant level leads to "ringing" of the current in response to an abrupt change of bias, and similarly complex time-dependence in response to an ac-bias. This "ringing" can be observed experimentally in the dc-current by varying the pulse length in a train of voltage pulses. We hope that time-dependence will provide a new window on coherent quantum transport and will lead to significant new insights in the future.

\section{ACKNOWLEDGMENTS}

We thank S. J. Allen, M. Kastner, and L. Kouwenhoven for valuable discussions. One of us (NSW) gratefully acknowledges the NORDITA mesoscopic program for hospitality during early stages of this work. Work at UCSB was supported by NSF grant no. NSF-DMR9001502 and by the NSF Science and Technology Center for Quantized Electronic Structures, 
Grant no. DMR 91-20007. 


\section{Figure Captions:}

(1a) Time-dependent current, $J(t)$, through a symmetric double-barrier tunneling structure in response to a rectangular bias pulse. All energies are in units of the elastic coupling to the leads, $\Gamma$, the current is in units of $e \Gamma / \hbar$, and all times are in units of $\hbar / \Gamma$. Initially, the chemical potentials $\mu_{L}$ and $\mu_{R}$ and the resonant-level energy $\epsilon_{0}$ are all zero. At $t=0$, a bias pulse (dashed curve) suddenly increases energies in the left lead by $\Delta_{L}=10$ and increases the resonant-level energy by $\Delta=5$ (see inset). At $t=3$, before the current has settled to a new steady value, the pulse ends and the current decays back to zero. By symmetry, the occupancy of the resonant level is always 0.5 and the currents through the two barriers are equal. The temperature in all figures is $k_{B} T=0.1 \Gamma$. (1b) Derivative of the integrated dc-current, $J_{\mathrm{dc}}$, with respect to pulse duration, $s$, normalized by the interval between pulses, $\tau$. For pulse durations much longer than the resonance lifetime $\hbar / \Gamma$, the derivative is just the steady-state current at the bias voltage, but for shorter pulses the "ringing" response of the current is evident.

(2a) Time-dependent current, $J(t)$, through a symmetric double-barrier tunneling structure for an ac-bias of frequency $\omega=2 \Gamma / \hbar$ (dashed curve). The ac-driving amplitude is $\Delta_{L}=10$ about $\mu_{L}=10$ in the left lead, $\Delta=5$ about $\epsilon_{0}=5$ for the level, and $\Delta_{R}=\mu_{R}=0$ in the right lead (see inset). By symmetry $n=0.5$ and the currents through the two barriers

are equal. $(2 \mathrm{~b})$ Time-averaged current, $J_{\mathrm{dc}}$, as a function of the ac-oscillation period $2 \pi / \omega$. The ac-amplitudes are the same as those used in (a). 


\section{References}

*Also at: MIC, Technical University of Denmark, DK-2800 Lyngby, Denmark

1. For a review see P. A. Lee and T. V. Ramakrishnan, Rev. Mod. Phys. 57, 287 (1985).

2. For a series of review articles see Mesoscopic Phenomena in Solids, B. L. Altshuler, P. A. Lee, and R. A. Webb, eds. (Elsevier, Amsterdam, 1991).

3. J. F. Whitaker et al., Appl. Phys. Lett. 53, 385 (1988); E. R. Brown et al., Appl. Phys. Lett. 58, 2291 (1991).

4. L. P. Kouwenhoven et al., Phys. Rev. Lett. 67, 1626 (1991); for a recent review see Single Charge Tunneling, H. Grabert, J. M. Martinis, and M. H. Devoret, eds. (Plenum, New York, 1991).

5. P. S. S. Guimaraes et al., preprint.

6. A linear-response formula for the time-dependent non-interacting current was recently derived by Y. Fu and S. C. Dudley, Phys. Rev. Lett. 70, 65 (1993).

7. The time-averaged current in response to an ac-bias has been analyzed previously for this model: D. Sokolovski, Phys. Rev. B 37, 4201 (1988); P. Johansson, Phys. Rev. B41, 9892 (1990). Johansson also considers the time-dependent current in a phenomenological approach.

8. Y. Meir and N. S. Wingreen, Phys. Rev. Lett. 68, 2512 (1992).

9. L. V. Keldysh, Sov. Phys. JETP 20, 1018 (1965); C. Caroli et al., J. Phys. C 4, 916 (1971).

10. G. D. Mahan, in Many-Particle Physics, edited by (Plenum Press, New York, 1990).

11. N. S. Wingreen, K. W. Jacobsen, and J. W. Wilkins, Phys. Rev. B 40, 11834 (1989).

12. K. L. Jensen and F. A. Buot, Phys. Rev. Lett. 66, 1078 (1991). 
13. The initial change in current (11) is due to a change in $A_{L / R}(\epsilon, t)$. The change, $\delta A_{L / R}(\epsilon, t)$, has an overall amplitude proportional to $t$, and a long tail $(\propto 1 / \epsilon)$ out to energies $|\epsilon| \sim \hbar / t$, which leads to a growth of the current as $t \int_{-\hbar / t}^{\mu} d \epsilon / \epsilon \sim t \log (1 / t)$.

14. L. Y. Chen and C. S. Ting, Phys. Rev. Lett. 64, 3159 (1990); Phys. Rev. B43, 2097 (1991).

15. Leo Kouwenhoven, private communication; however, to observe the "ringing" experimentally requires a pulse rise-time faster than the ringing period. 